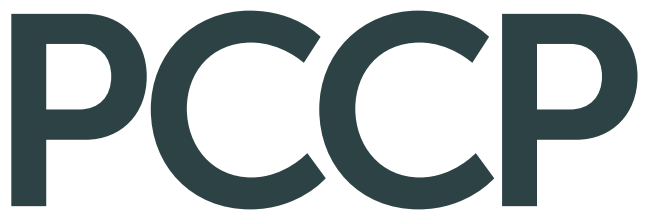

Physical Chemistry Chemical Physics rsc.li/pccp

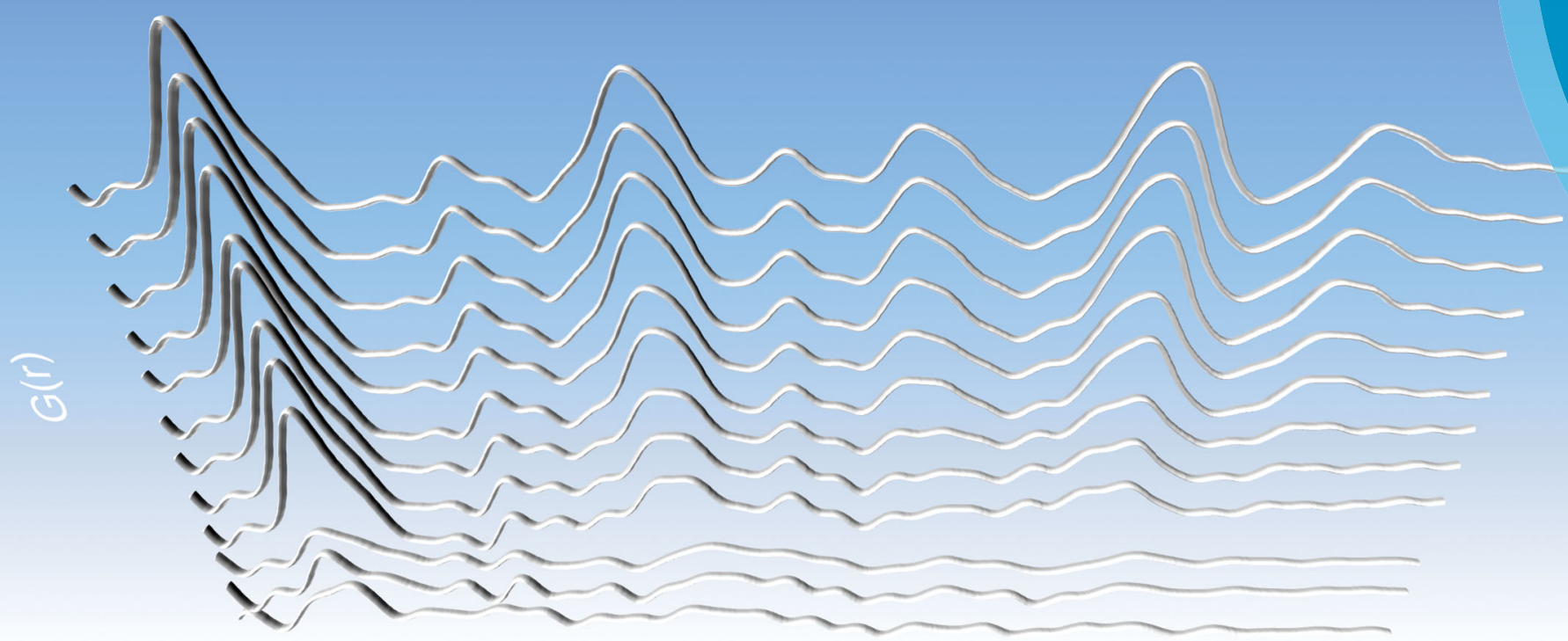

2

4

6
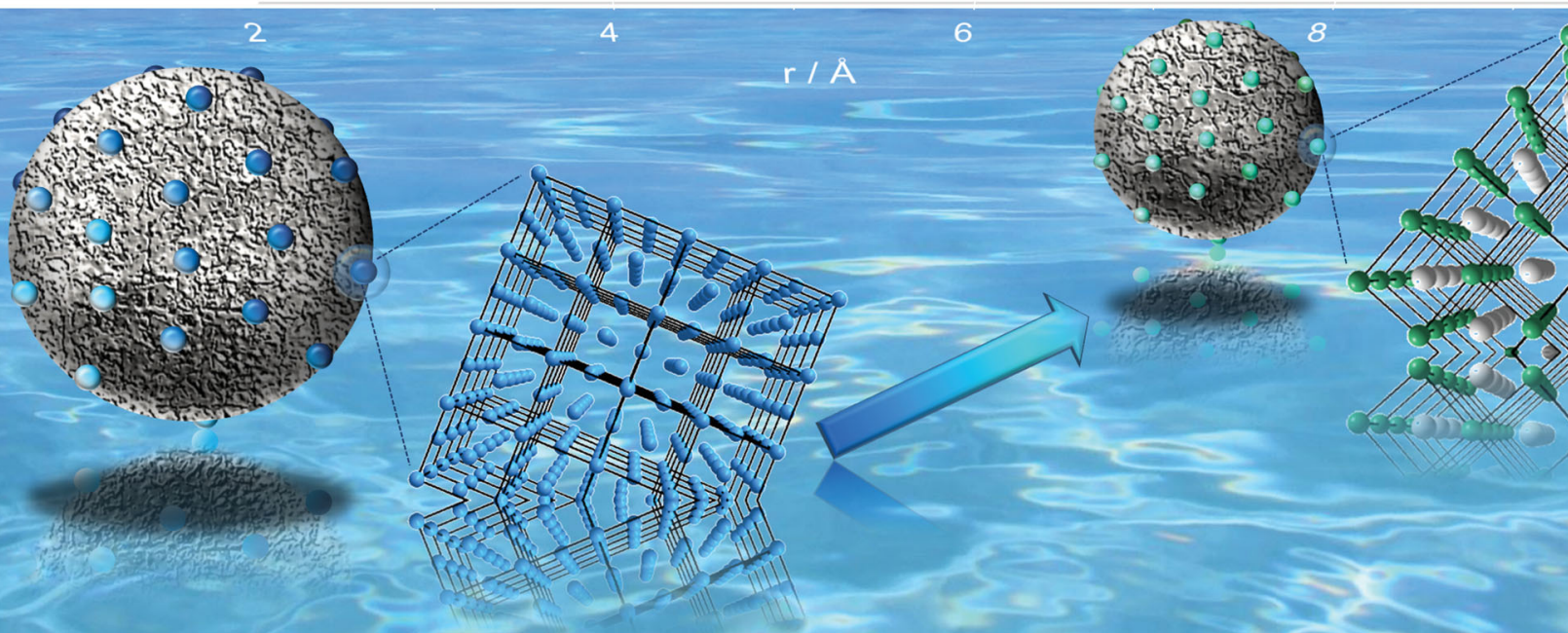
Check for updates

Cite this: Phys. Chem. Chem. Phys., $2018,20,8466$

\title{
Monitoring the formation of PtNi nanoalloys supported on hollow graphitic spheres using in situ pair distribution function analysis $\dagger$
}

\author{
Ş. Ortatatlı, (D) J. Knossalla, F. Schüth and C. Weidenthaler (DD *
}

\begin{abstract}
This article aims to address the formation and the structural disordering/ordering phenomena of PtNi nanoalloys supported on hollow graphitic spheres (HGSs) using pair distribution function (PDF) analysis under ex situ/in situ data collection conditions. Starting from small nanoparticles (10-15 $\AA$ in diameter) embedded in HGSs, structural changes were monitored during stepwise heating and cooling of the sample using in situ PDF analysis. In order to evaluate the conventional synthesis route for the production of PtNi nanoalloys supported on HGSs, ex situ PDF experiments were performed before and after heat treatment in a furnace. The studies demonstrate that the local structure of the in situ synthesised PtNi nanoalloy differs from its ex situ synthesised counterpart. A partially ordered PtNi nanoalloy was obtained during the stepwise in situ cooling of the precursor, whereas the conventional ex situ synthesis route did not lead to the formation of an ordered crystal structure. In this study we could show that rapid heating and cooling results in a disordered PtNi alloy whereas slow heating and cooling leads to disorder-order transitions in PtNi.
\end{abstract}

Received 21st November 2017

Accepted 3rd January 2018

DOI: $10.1039 / c 7 c p 07840 d$

rsc.li/pccp in the synthesis routes result in different, partly contradictory conclusions regarding the structure-catalytic activity relationship. In situ structure characterisation could help here, since this provides direct insights into structural changes. ${ }^{15,28-32}$ Especially disorder/order phenomena and their influence on the ORR have been discussed in the past. For PtNi nanoparticles, a significantly lower ORR activity was observed for disordered PtNi alloys. ${ }^{18}$ In this regard, pair distribution function (PDF) analysis is a powerful technique, as not only the local structure, but also the long range order (LRO) structure can be detected with high accuracy. ${ }^{33,34}$ There are few in situ PDF studies to bridge the gap between structure and electrocatalytic activity enhancement, as well as to monitor structural changes under operating conditions of PEMFCs. ${ }^{15,28-31}$ Among them Redmond et al. were the first to apply the PDF method to follow the particle growth of Pt nanoparticles supported on carbon in an operating fuel cell. ${ }^{30}$ However, to the best of the authors' knowledge, fundamental investigations on the formation of PtNi nanoalloys have not been reported yet from the in situ $\mathrm{X}$-ray total scattering viewpoint. Despite the investigations on the transition temperature from a disordered to ordered structure of a bulk material, ${ }^{35,36}$ the experimental results cannot be directly transferred to PtNi nanoparticles because the physicochemical properties of a bulk material may differ significantly from its nanosized counterpart. In this paper we aim to reveal the formation and disordering/ordering phenomena of PtNi nanoalloys supported on hollow graphitic spheres (HGSs) using in situ X-ray PDF analysis in combination with conventional X-ray powder diffraction (XRPD). 


\section{Experimental}

\section{Synthesis}

Hollow graphitic spheres (HGSs). HGSs are porous carbon materials used as supports in electrocatalysis. The synthesis of HGSs has already been described elsewhere. ${ }^{27,37,38}$ Briefly, $1 \mathrm{~g}$ of the silica exotemplate (with a total pore volume of $0.37 \mathrm{~cm}^{3} \mathrm{~g}^{-1}$ determined by $\mathrm{N}_{2}$-physisorption, not shown here) was impregnated twice with an ethanolic solution of $\mathrm{Fe}\left(\mathrm{NO}_{3}\right)_{3} \cdot 9 \mathrm{H}_{2} \mathrm{O}, 2 \mathrm{M}(0.37 \mathrm{~mL}$ each). The slurry was dried for $12 \mathrm{~h}$ at $75{ }^{\circ} \mathrm{C}$. Prior to the impregnation of the polymer precursor, the silica exotemplate/catalyst material was evacuated under vacuum for $3 \mathrm{~h}$. Afterwards, a mixture of $0.37 \mathrm{~mL}$ of divinylbenzene (DVB) and $0.02 \mathrm{~g}$ azo-bis-(isobutyronitrile) (AIBN) was incorporated into the mesopores of the silica exotemplate under Ar flow. The resulting material was heated at $75{ }^{\circ} \mathrm{C}$ for $24 \mathrm{~h}$ to complete the polymerisation of DVB under Ar. The carbonisation of poly(divinylbenzene) (PDVB) in the mesopores was achieved by thermal treatment to $1000{ }^{\circ} \mathrm{C}$ (with a heating rate of $5{ }^{\circ} \mathrm{C} \mathrm{min}^{-1}$ ) for $4 \mathrm{~h}$ under $\mathrm{N}_{2}$ flow. The silica exotemplate and iron residuals were removed by subsequent $\mathrm{HF}$ and $\mathrm{HCl}$ acid treatments, respectively. Finally, the material was washed and dried at $75{ }^{\circ} \mathrm{C}$ overnight.

Ex situ synthesis of PtNi nanoparticles. The synthesis of PtNi nanoparticles using the confined space alloying method was described elsewhere. ${ }^{14,26}$ Typically, PtNi nanoparticles were embedded in HGSs via an impregnation-reduction-heating procedure as illustrated in Fig. 1.

Aqueous metal chloride solutions, namely $\mathrm{H}_{2} \mathrm{PtCl}_{6} \cdot x \mathrm{H}_{2} \mathrm{O}$ (Pt (wt\%): $39 \pm 1$ ) and $\mathrm{NiCl}_{2} \cdot 6 \mathrm{H}_{2} \mathrm{O}$, were introduced into the pores of the as-synthesised HGSs through an ultrasound assisted incipient wetness impregnation. The total volume of the resulting solution was adjusted with deionised ultrapure water to reach $90 \%$ of the carbon's total pore volume. After the impregnation, the wet powder was ultrasonicated for $30 \mathrm{~min}$, and the solvent was removed holding the wet material under Ar for $30 \mathrm{~min}$ at $120{ }^{\circ} \mathrm{C}$ (heating rate $3{ }^{\circ} \mathrm{C} \mathrm{min}{ }^{-1}$ ). The reduction of the metal precursors was performed in $\mathrm{a}_{2} / \mathrm{Ar}$ gas mixture $\left(15\right.$ vol\% $\mathrm{H}_{2}$ ) at $220{ }^{\circ} \mathrm{C}$ for $90 \mathrm{~min}$. Afterwards the $\mathrm{H}_{2}$ flow was switched off, and the sample was left to cool to room temperature. In the final step of the ex situ synthesis, the sample was heated to $850{ }^{\circ} \mathrm{C}$ (heating rate $5{ }^{\circ} \mathrm{C} \mathrm{min}^{-1}$ ) in a tube furnace under continuous Ar flow and held at this temperature for $6 \mathrm{~h}$. Ex situ synthesised PtNi (after step 3, Fig. 1) was investigated via an ex situ total scattering technique, while the reduced sample,

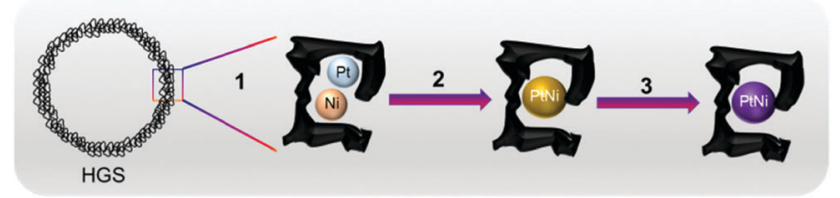

Fig. 1 Schematic illustration for the preparation of PtNi nanoalloys supported on HGSs using an incipient wetness impregnation method: (1) incipient wetness impregnation of the as-synthesised HGS support with metal chloride solutions, (2) subsequent reduction with $\mathrm{H}_{2}$ at $220{ }^{\circ} \mathrm{C}$ to obtain PtNi precursor used for in situ total scattering experiments, (3) heating at $850{ }^{\circ} \mathrm{C}$ in Ar to obtain crystalline PtNi nanoalloys used for ex situ total scattering experiments. i.e., the precursor (after step 2, Fig. 1) was used directly for the in situ total scattering experiments.

\section{Characterisation}

In situ total scattering data collection. In situ total scattering experiments were performed at the European Synchrotron Radiation Facility (ESRF, ID22 high resolution powder diffraction beamline). A monochromatic X-ray beam was used ( $\lambda: 0.2065474 \AA)$. The data were acquired at $60 \mathrm{keV}$. A two-dimensional flat panel Perkin-Elmer detector was used for the data acquisition. The detector to sample distance was $380 \mathrm{~mm}$. A maximum scattering vector $\left(Q_{\max }\right)$ of $25 \AA^{-1}$ was achieved. A hot air blower mounted on a moving table was connected to the setup for heating of the precursor. The experimental setup used for in situ total scattering experiments at the ID22 beamline can be found in the ESI $\dagger$ (Section 1, Fig. S1). Since in situ experiments were performed under atmospheric conditions, the PtNi precursor (after step 2, Fig. 1) was sealed into a quartz glass capillary $(0.5 \mathrm{~mm} \varnothing)$ in order to prevent self-ignition and combustion of the HGS support. Stepwise heating of the precursor was performed from 25 to $800{ }^{\circ} \mathrm{C}$ with a heating rate of $5{ }^{\circ} \mathrm{C} \mathrm{min}{ }^{-1}$, and data were collected at selected temperatures for $45 \mathrm{~min}$ (temperature program is given in the ESI, $\dagger$ Section 2, Fig. S2). Data were collected during stepwise cooling for $45 \mathrm{~min}$ at each selected temperature as well. Due to restriction of the experimental time, cooling of the sample from $400{ }^{\circ} \mathrm{C}$ to room temperature could not be completed. The HGS support was measured in the same type of capillary for the background subtraction in the PDF. Data calibration and integration were performed with the Fit2D program ${ }^{39}$ using LaB $_{6}$ (NIST-standard) as a calibration sample.

Ex situ total scattering data collection. Ex situ total scattering experiments were performed at the Diamond Light Source (DLS, I15-Extreme Conditions beamline). A monochromatic X-ray beam $(\lambda: 0.1721616 \AA)$ and a two-dimensional flat panel Perkin-Elmer detector were used. Data were acquired at $72 \mathrm{keV}$. The detector to sample distance was $206.88 \mathrm{~mm}$. A maximum scattering vector $\left(Q_{\max }\right)$ of $27 \AA^{-1}$ was achieved. The experimental setup used at the I15 beamline is shown in the ESI, $\dagger$ (Section 3, Fig. S3). The PtNi precursor (after step 2, Fig. 1) and the ex situ synthesised crystalline PtNi nanoalloy (after step 3, Fig. 1) were filled into borosilicate glass capillaries $(0.5 \mathrm{~mm} \varnothing)$. The HGS support in the same type of capillary was measured as well for the background subtraction in the PDF. Data calibration and integration were done with Fit2D ${ }^{39}$ using $\mathrm{CeO}_{2}$ (NIST-standard) as the calibration sample.

High angle annular dark field scanning transmission electron microscopy (HAADF-STEM). Dry prepared PtNi powder before and after heat treatment was analysed on a regular single tilt holder by HAADF imaging using the Hitachi HD-2700 Cs-corrected $200 \mathrm{kV}$ dedicated STEM, equipped with an EDAX Octane T Ultra W EDSdetector.

Data analysis and structure visualisation. In situ/ex situ high resolution (HR) XRPD data were analysed using the TOPAS Academic version 4.2 program, ${ }^{40}$ while in situ/ex situ PDF data were generated and fitted by using the PDFgetX3 and the PDFgui programs, respectively. ${ }^{41,42}$ Instrument dependent parameters, 
i.e., $Q_{\mathrm{damp}}$ and $Q_{\mathrm{broad}}$, were determined by fitting the in situ and ex situ PDF data of $\mathrm{LaB}_{6}$ (NIST-standard) and $\mathrm{CeO}_{2}$ (NIST-standard), respectively. The Diamond program was used to visualise the crystal structures and calculate the corresponding interatomic distances as well as the XRPD patterns. ${ }^{43}$

\section{Results and discussion}

In Fig. 2 the simulated crystal structures of ordered and disordered PtNi and their corresponding calculated XRPD patterns are shown. The room temperature crystallographic data of disordered and ordered PtNi were taken from the Inorganic Crystal Structure Database (ICSD): 105317 and 646297, respectively. ${ }^{35,44}$ Disordered PtNi crystallises in a cubic structure $(a=3.7499$ (1) Å; space group: $F m \overline{3} m$, Wyckoff position (Pt, Ni) = 4a) (Fig. 2a), while its ordered counterpart crystallises in a tetragonal structure $(a=2.7019$ (1) A, $c=3.5909$ (1) Å; space group: P4/mmm, Wyckoff position of Pt: $1 \mathrm{~d}$ and Ni: 1a) (Fig. 2b). In the case of a disordered PtNi structure, Pt and $\mathrm{Ni}$ atoms are situated at $(0,0,0)$ and are randomly distributed over the unit cell with equal probability (Fig. 2a, inset unit cell). In the case of an ordered structure, Pt is situated at $(1 / 2,1 / 2,1 / 2)$ in the center, while $\mathrm{Ni}$ atoms are located at $(0,0,0)$ on the corners of the unit cell (Fig. 2b, inset unit cell). The calculated XRPD patterns show that the ordered structure differs from its disordered counterpart by some additional reflections, i.e., the so-called superstructure reflections (Fig. 2b).

\section{In situ formation of PtNi nanoalloy-heating: Rietveld analysis}

Fig. 3 shows in situ HR-XRPD data recorded during heating, plotted on the $Q(\AA)$ scale. The reflection at $\sim 1.85 \AA(Q)$ is attributed to the graphitic support (ICSD-53781). ${ }^{45}$ Indexed reflections in Fig. 3 belong to a pure disordered PtNi alloy.

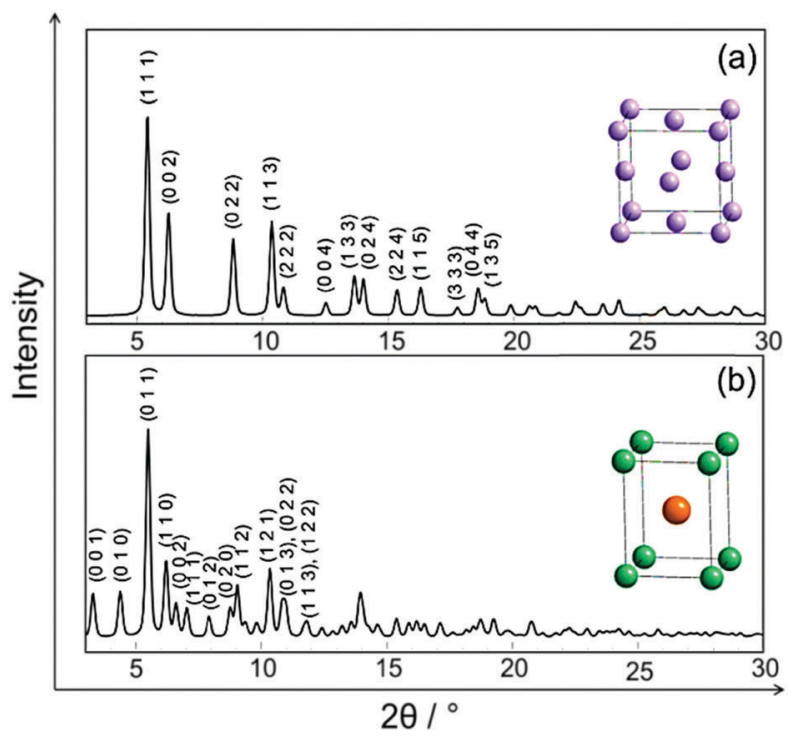

Fig. 2 Calculated XRPD patterns for $\lambda=0.2065474 \AA$ and crystal structures (inset) of PtNi alloys: (a) disordered, (b) ordered. The colour code of the atoms: disordered PtNi (O), Pt (O) and $\mathrm{Ni}(\mathrm{O})$. Numbers in parentheses indicate Miller indices.
At low temperatures, the reflections can hardly be seen due to a small domain size $(\sim 10-15 \AA$ in diameter $)$ of the precursor (Fig. 3). Significant structural changes are observed in the temperature range between 400 and $800{ }^{\circ} \mathrm{C}$ (Fig. 3).

Rietveld analyses of in situ HR-XRPD data recorded at 700, 750 and $800{ }^{\circ} \mathrm{C}$ verify the formation of a bulk crystalline disordered PtNi nanoalloy upon heating. Details regarding the Rietveld analysis procedure and the analysis results are provided in the ESI, $\dagger$ (Section 4.1) as well as the Rietveld plots of the data recorded at 700 and $750{ }^{\circ} \mathrm{C}$ (Section 4.1, Fig. S4 and Table S1, ESI $\dagger$ ). In Fig. 4 Rietveld analysis of the data recorded at $800{ }^{\circ} \mathrm{C}$ is shown. The data were fitted against a disordered PtNi model. A good agreement is observed between the model and the experimental data (Fig. 4). The unit cell parameter (a) of 3.7789 (1) A obtained after fitting deviates significantly from the unit cell parameter before fitting ( $a=3.7499$ (1) $\AA$ ). This is explained by the linear thermal expansion $\left(\alpha=0.9978 \times 10^{-5} \mathrm{C}^{-1}\right)$ of the unit cell at high temperature $\left(800{ }^{\circ} \mathrm{C}\right)$.

\section{In situ formation of PtNi nanoalloy-heating: PDF analysis}

Fig. 5 shows in situ PDF data collected during heating. Data collected at 25,250 and $300{ }^{\circ} \mathrm{C}$ do not show the features of a crystalline precursor, as the peaks are relatively broad and not well-defined. This might be related to the amorphous state and/ or small domain size $(\sim 10-15 \AA)$ of the precursor (Fig. 5). With increasing temperature PDF peaks terminate at longer distances implying particle growth (Fig. 5a). For example, the peak cut-offs determined for the data collected at 25,250 and $300{ }^{\circ} \mathrm{C}$ correspond to the diameter of an ordered domain of $\sim 10 \AA$ which is in accordance with the HAADF-STEM analysis results (shown in Fig. 15, vide infra), whereas the peak cut-off at $800{ }^{\circ} \mathrm{C}$ is observed around $\sim 50 \AA$ (Fig. 5a). This is a proof for particle growth. The peak intensities and the peak widths change remarkably during heating (Fig. $5 \mathrm{~b}$ ). To be more specific, the first PDF peak measured at $400{ }^{\circ} \mathrm{C}$ is much sharper than those measured at 25,250 and $300{ }^{\circ} \mathrm{C}$. This is probably caused by successive crystallisation of the precursor upon heating (Fig. 5b).

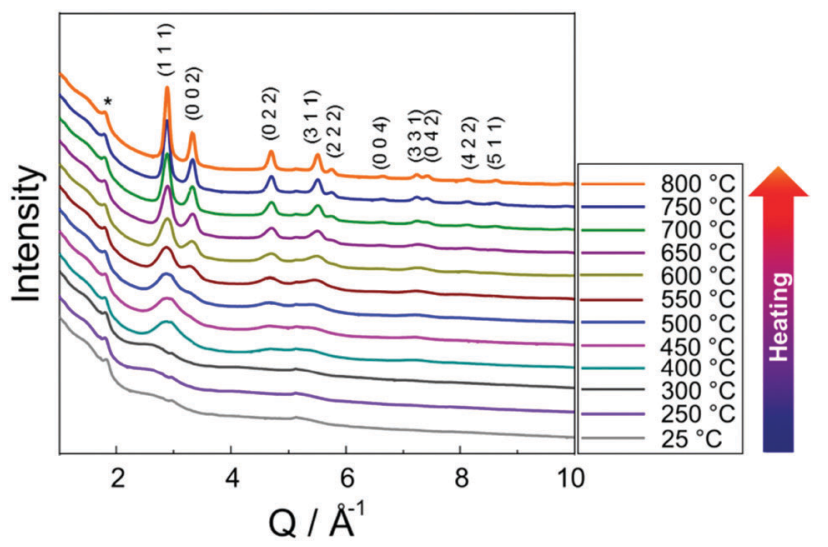

Fig. 3 In situ HR-XRPD data of the PtNi precursor recorded during stepwise heating. The indexed reflections belong to the data recorded at $800{ }^{\circ} \mathrm{C}$. The asterisk symbol (*) is used to highlight carbon reflection (HGS support). 


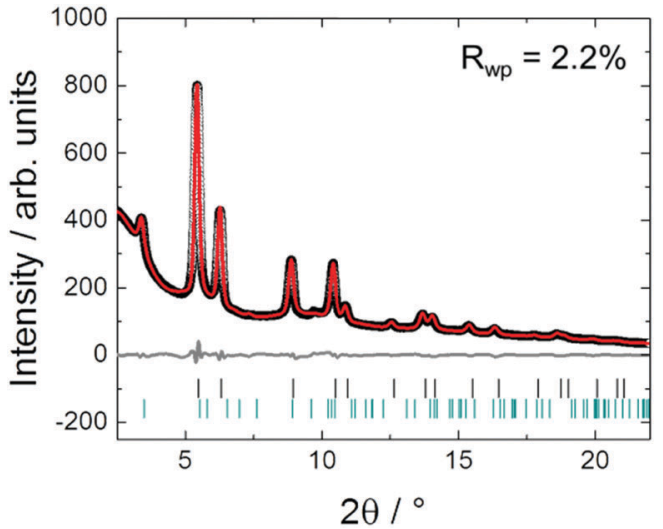

Fig. 4 Rietveld analysis of the in situ HR-XRPD data of PtNi on HGSs recorded at $800{ }^{\circ} \mathrm{C}$. The black circles represent the experimental data, while the red and the grey curves represent the calculated data and the difference, respectively. Tick marks denote the calculated peak positions of disordered PtNi (black) and carbon (green). $R_{\mathrm{wp}}$ is the fit residual.

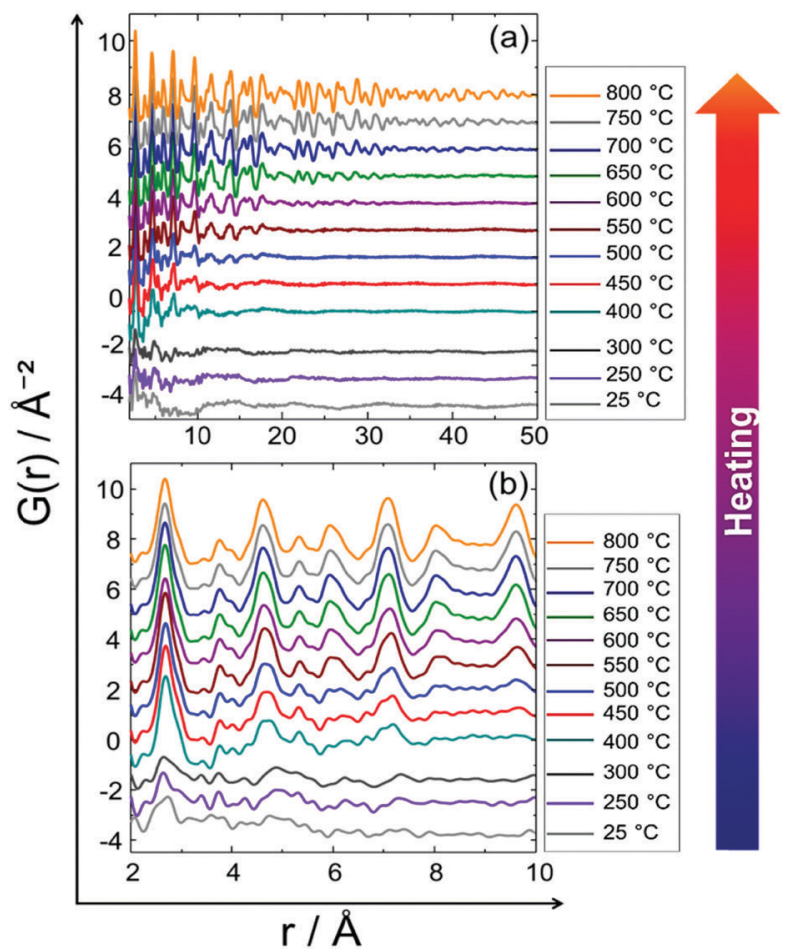

Fig. 5 In situ PDF data of the PtNi precursor collected upon heating from room temperature to $800{ }^{\circ} \mathrm{C}$ : (a) long range order (LRO), (b) short range order (SRO)

As partial PDF calculations provide beneficial information about the atom pairs contributing to an experimental PDF, the local structures of the precursor at 25,250 and $300{ }^{\circ} \mathrm{C}$ were analysed by comparison to the calculated partial PDFs (ESI, $\uparrow$ Section 4.2, Fig. S5). The real space resolution ( $\delta r)$ of the in situ PDF data is $0.13 \AA$ (calculated by $\pi / Q_{\max }$ ). As a result, severe peak overlap is observed in the experimental PDF data (Fig. 5). For example, the first theoretical Pt-Pt distance is $2.77 \AA$ in a pure metallic Pt structure, while the first $\mathrm{Ni}-\mathrm{Ni}$ distance is
$2.49 \AA$ in a pure metallic Ni and the first PtNi-PtNi distance is $2.65 \AA$ in a disordered PtNi structure. Since $\delta r$ is $0.13 \AA$, these three interatomic distances cannot be resolved but overlapped instead, giving rise to peak envelopes (Fig. 5b and Fig. S5, ESI $\dagger$ ). Nonetheless, the experimental and the calculated partial PDF peak positions in Fig. S5 (ESI $\dagger$ ) suggest that after the reduction step at $220{ }^{\circ} \mathrm{C}$ during the synthesis procedure, the precursor is likely a disordered PtNi with some concomitant metallic Ni and $\mathrm{Pt}$ at room temperature.

A list of the theoretical and the experimental interatomic distances of disordered PtNi at 25 and $800{ }^{\circ} \mathrm{C}$ is provided in the ESI, $\dagger$ (Section 4.2, Table S2). The theoretical distances were calculated based on a disordered PtNi model. ${ }^{35}$ In order to analyse the local structure at $800{ }^{\circ} \mathrm{C}$, PDF fitting was performed using the least squares approach in the PDFgui program (Fig. 6). The data were fitted against a disordered PtNi model. Details regarding the PDF fitting are given in the ESI, $\dagger$ (Section 4.2). The unit cell parameter of 3.788 (2) Å obtained after fitting is larger than the starting unit cell parameter $(a=3.7499$ (1) $\AA$ ) due to thermal expansion. Also the $U_{\text {iso }}$ parameter is quite large after fitting, i.e., 0.024 (1) $\AA^{-2}$, which might be explained by temperature induced dynamic disorder. Nonetheless, the PDF fit in Fig. 6 shows a good agreement between the disordered model and the experimental data. The disordered model thus best reflects the true local structure of PtNi nanoalloys at $800{ }^{\circ} \mathrm{C}$.

\section{In situ formation of PtNi nanoalloy-cooling: Rietveld analysis}

Stepwise cooling of the PtNi precursor from 750 to $400{ }^{\circ} \mathrm{C}$ is shown in Fig. 7. It is evident that ordering of the structure starts during cooling as the additional low-intensity reflections appear at $550{ }^{\circ} \mathrm{C}$ at $2.35,3.66$ and $4.16 \AA(Q)$ and remain stable upon cooling to $400{ }^{\circ} \mathrm{C}$. These are the superstructure reflections indicating the formation of an ordered structure.

Although the superstructure reflections of the ordered phase appear during cooling, two different models were used to approximate the true crystal structure of PtNi nanoalloys by

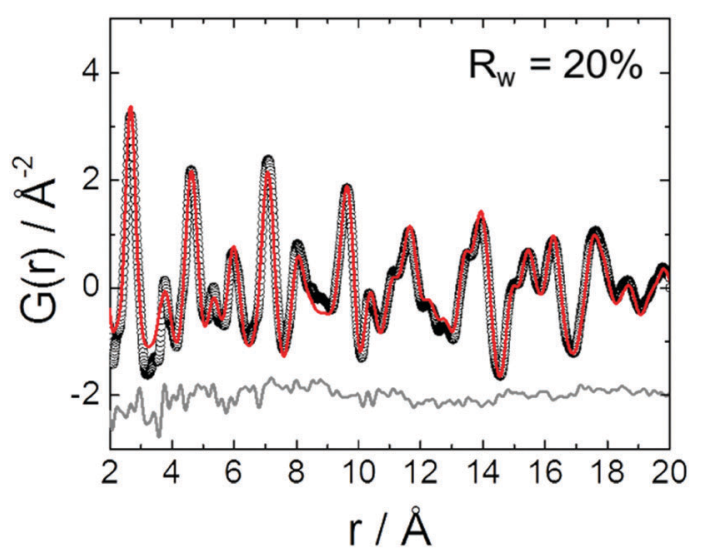

Fig. 6 PDF fit of the in situ data collected at $800{ }^{\circ} \mathrm{C}$ against a disordered PtNi model. The black circles represent the experimental data, whereas the red and the grey curves represent the calculated data and the difference, respectively. $R_{\mathrm{w}}$ is the PDF fit residual. 


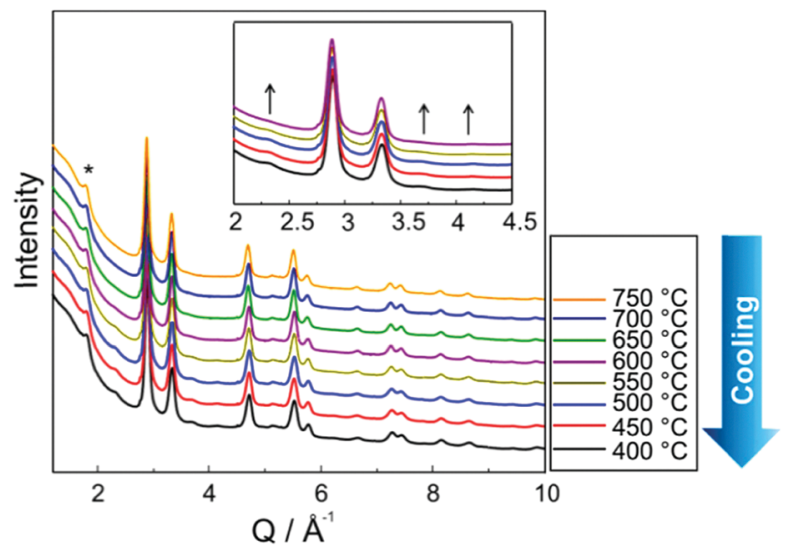

Fig. 7 In situ HR-XRPD data of PtNi on HGSs recorded during stepwise cooling. Asterisk symbol $(*)$ is used to highlight carbon reflection (HGS support), whereas the arrows (inset) are guide to eye indicating the superstructure reflections.

Rietveld analysis of the data recorded during cooling at $400{ }^{\circ} \mathrm{C}$ (Fig. 8): (1) fully ordered, (2) partially ordered. The latter is defined as a combination of ordered and disordered phases in this paper. The Rietveld analysis procedure and the analysis results are given in the ESI, $\uparrow$ (Section 5.1, Table S3). It is evident in Fig. 8a that the bulk crystal structure is not fully ordered

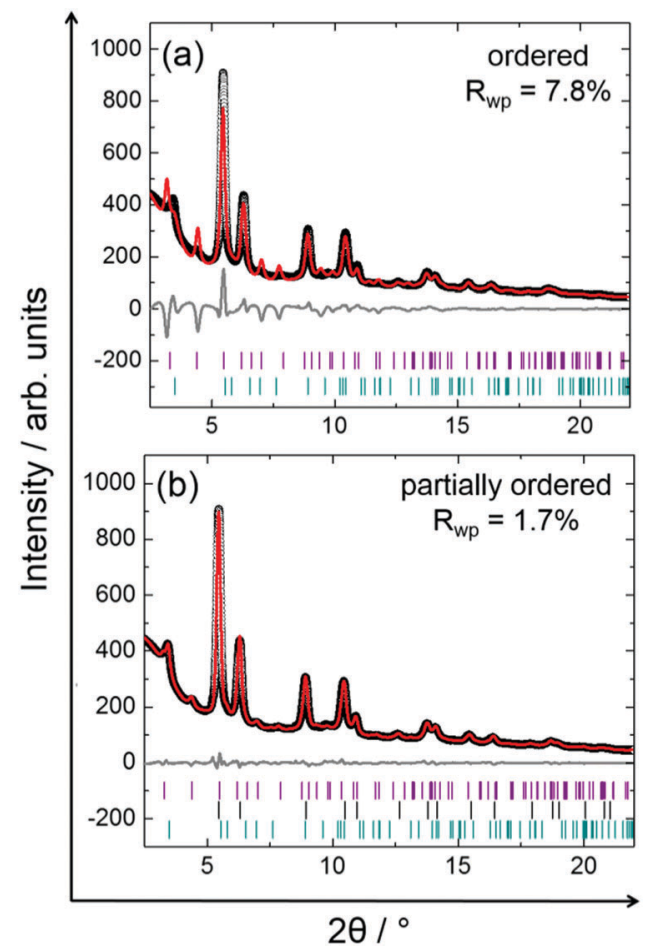

Fig. 8 Rietveld analyses of the in situ HR-XRPD data recorded at $400{ }^{\circ} \mathrm{C}$ during cooling using two different models: (a) ordered, (b) partially ordered. The black circles represent the experimental data, while the red and the grey curves represent the calculated data and the difference, respectively. Tick marks denote the calculated peak positions of disordered PtNi (black), ordered PtNi (purple), and carbon (green). $R_{w p}$ is the fit residual. because a severe discrepancy is observed between the model and the experimental data. The fit residual $R_{\mathrm{wp}}$ reduces significantly from $7.8 \%$ to $1.7 \%$ with the partially ordered model as the number of fit parameters increases by the combination of the ordered and disordered models (Fig. 8a).

\section{In situ formation of PtNi nanoalloy-cooling: PDF analysis}

Fig. 9 shows the experimental in situ PDF data collected during cooling. The PDF peak cut-off range is nearly the same in all patterns (Fig. 9a). This indicates that the particles do not grow further during cooling (Fig. 9a). Moreover, the peak intensities increase from 750 to $400{ }^{\circ} \mathrm{C}$ which is an indicator of the formation of a well-defined, crystalline, local structure. Short range order (SRO) PDFs, especially the first nearest neighbour distance shows the significant changes in the peak width and intensity more clearly (Fig. 9b).

Partial PDFs were calculated also for the PDF data collected during cooling and are shown in the ESI, $\dagger$ (Section 5.2, Fig. S6). According to the calculated partial PDFs, the experimental PDF recorded at $400{ }^{\circ} \mathrm{C}$ most probably consists of contributions from both disordered and ordered PtNi phases. In Table S4 (ESI, $\dagger$ Section 5.2) the theoretical and the experimental interatomic distances of ordered and disordered PtNi alloys are listed. The theoretical interatomic distances of disordered PtNi alloy are relatively close to the experimental distances given in Table S4 (ESI $\dagger$ ). However, in situ HR-XRPD data and Rietveld analysis unambiguously show the formation of the low-intensity superstructure reflections and partial ordering of the structure during cooling. For this reason, it is a reasonable

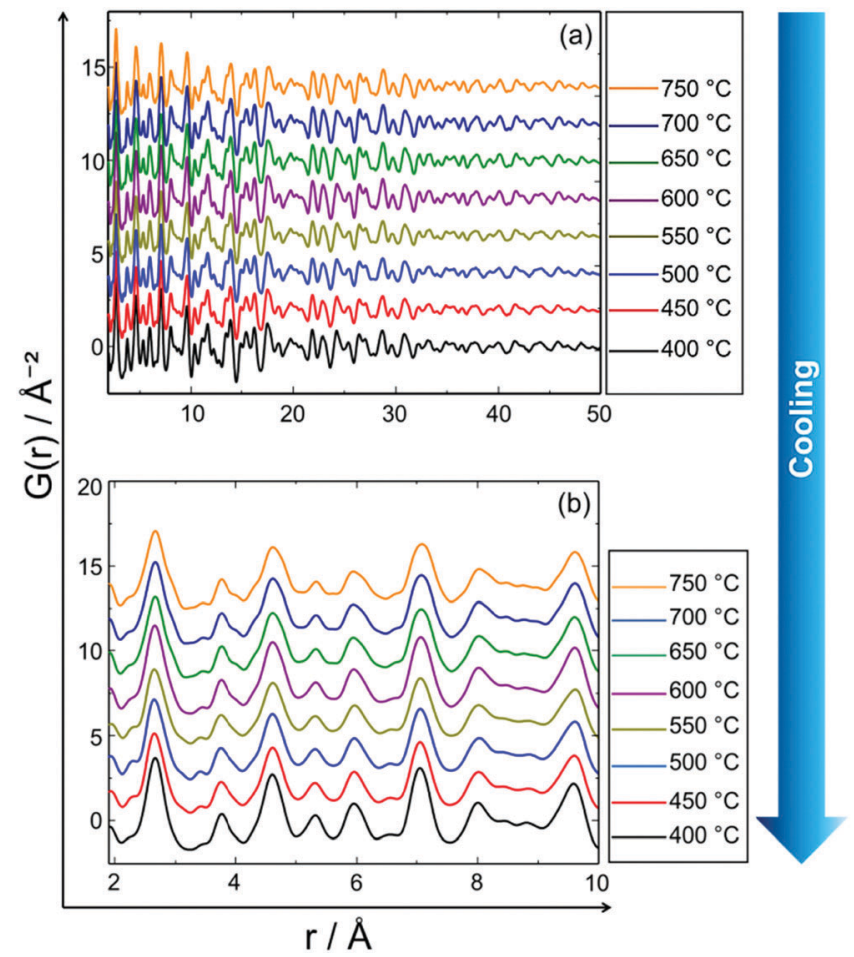

Fig. 9 In situ PDF data of PtNi recorded during stepwise cooling from $750{ }^{\circ} \mathrm{C}$ to $400{ }^{\circ} \mathrm{C}$ : (a) long range order (LRO), (b) short range order (SRO). 


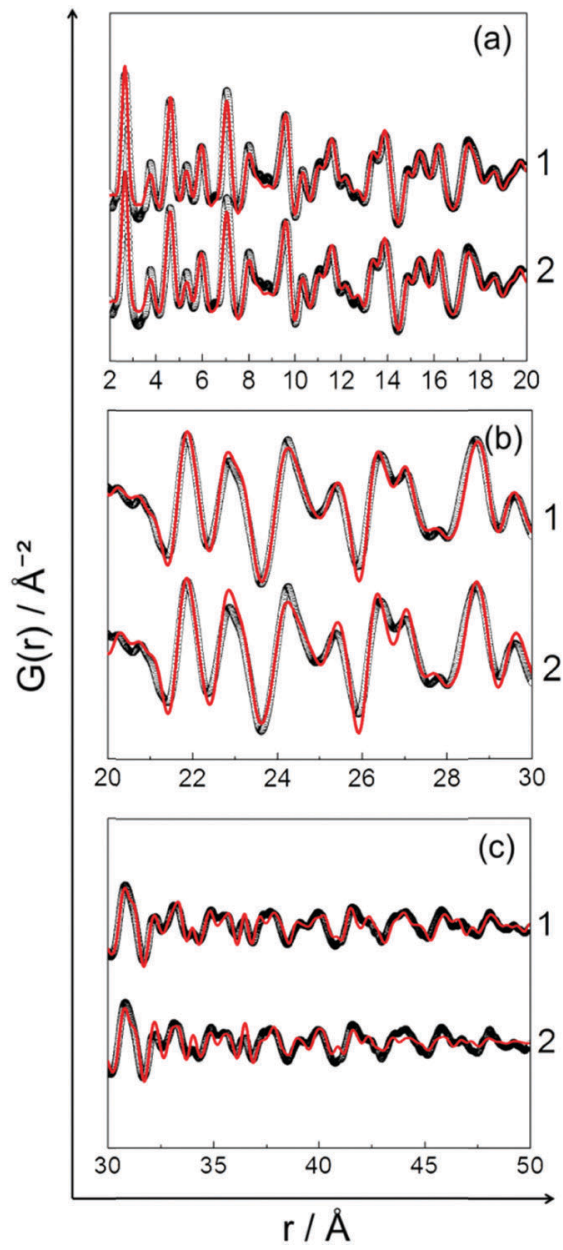

Fig. 10 Comparison of the $400{ }^{\circ} \mathrm{C}$-PDF fits using two different models: (a) short range order (SRO), (b) and (c) long range order (LRO). Numbers on the right side of the graphs are attributed to the models used for the fits: (1) partially ordered, (2) disordered. The black circles represent the experimental data, whereas the red curve represents the calculated data. Difference curves are not shown for the sake of clarity.

approximation to fit the PDF data collected during cooling at $400{ }^{\circ} \mathrm{C}$ against two different models: (1) partially ordered (ordered + disordered), (2) disordered. Fig. 10 shows the PDF fits using those models, while the results of the fits are provided in the ESI, $\dagger$ (Section 5.2, Table S5). Although the SRO-PDF fits look similar (Fig. 10a), a closer look to LRO-PDF fits in Fig. 10b and $\mathrm{c}$ shows that the disordered model does not reflect the true structure of PtNi nanoalloys. Significant discrepancies are observed between the calculated and the experimental data (Fig. 10b and c). On the other hand, the partially ordered model reflects the local structure of the formed nanoalloy from SRO to LRO. The increasing number of fit parameters results in a better fit (Fig. 10).

In Fig. 11 in situ HR-XRPD and PDF data collected during heating and cooling are compared. It is obvious that not only LRO but also SRO changes. The superstructure reflections, i.e., the indicators of an ordered PtNi crystal structure are visible at $400{ }^{\circ} \mathrm{C}$ after cooling, whereas they are not visible at $800{ }^{\circ} \mathrm{C}$ (Fig. 11a). Moreover, PDF peaks are much sharper and more
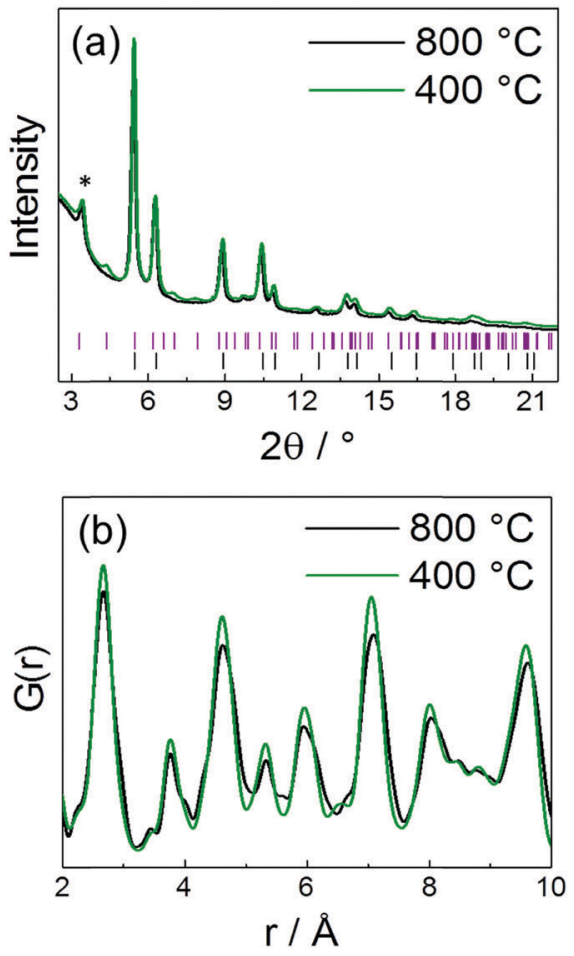

Fig. 11 Comparison of the in situ data collected during heating at $800{ }^{\circ} \mathrm{C}$ versus cooling at $400{ }^{\circ} \mathrm{C}$ : (a) HR-XRPD, (b) PDF. Tick marks in (a) represent the calculated peak positions of ordered PtNi (purple) and disordered PtNi (black). Symbol (*) in (a) highlights carbon reflection (HGS support).

intense after cooling to $400{ }^{\circ} \mathrm{C}$ than obtained at $800{ }^{\circ} \mathrm{C}$. This is a result of ordering of the local structure during cooling (Fig. 11b).

Based on the results obtained from in situ Rietveld and PDF analyses it is evident that the average bulk and the local structure of PtNi nanoalloys is disordered during heating, whereas partial ordering was achieved only during cooling (Fig. 12).

$\mathrm{XRD}$ and PDF analyses reveal that the transition of the PtNi crystal structure from a disordered to an ordered nanoalloy is a matter of time applied for the temperature treatment. The slower the cooling, the more ordered is the crystal structure.

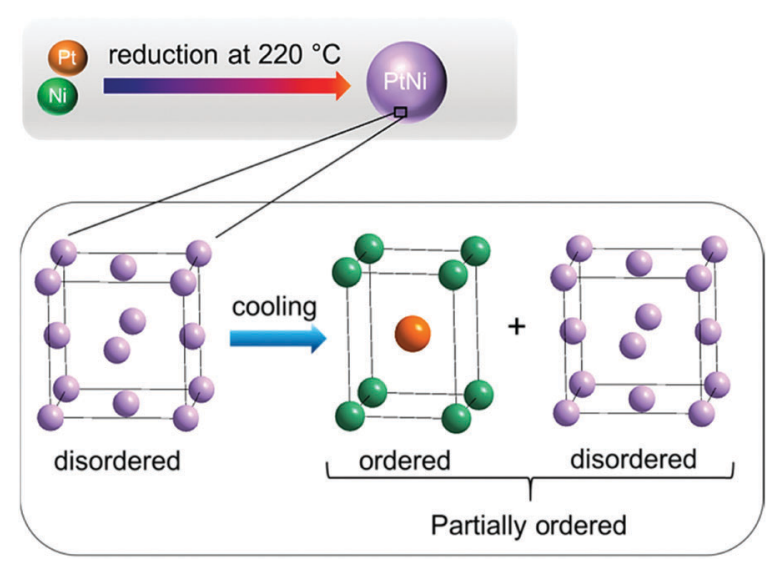

Fig. 12 Schematic illustration of PtNi nanoalloy formation: structure remains disordered during heating, whereas it is partially ordered during cooling. 
This is in accordance with the work carried out by Woolley and Bates in $1960 .^{36}$ They obtained a disordered PtNi alloy by fast cooling, i.e., quenching the sample in water after heating at $1000{ }^{\circ} \mathrm{C}$. In order to achieve ordering, they performed slow cooling of disordered PtNi alloy to room temperature. A more recent paper about the conversion of a PtNi alloy from a disordered to an ordered structure was published by Zou et al. in 2015. ${ }^{18}$ According to their work, after heating of disordered PtNi from 550 to $600{ }^{\circ} \mathrm{C}$ for different time periods an ordered PtNi nanoalloy was synthesised. However, neither the actual temperature program nor the heating time was mentioned in the paper. In addition, cooling was not reported at all. ${ }^{18}$

\section{Ex situ formation of PtNi nanoalloys: Rietveld analysis}

In Fig. 13 the Rietveld plot of ex situ HR-XRPD obtained for the sample after heating in a tube furnace to $850{ }^{\circ} \mathrm{C}$ and subsequent cooling is shown. The experimental data were fitted against a disordered PtNi model (Fig. 13). The fit in Fig. 13 indicates that the precursor undergoes different ordering mechanisms when heated and cooled in a tube furnace compared to its in situ synthesised counterpart because the superstructure reflections are not visible. This might be due to the fact that cooling in the tube furnace was faster $(<6 \mathrm{~h})$ than in situ cooling $(>20 \mathrm{~h})$ because of the in situ interval measurements at selected temperatures. The unit cell parameter of the disordered PtNi phase after fitting of $a=3.7505$ (7) A is within the standard deviation of the unit cell parameter before fitting ( $a=3.7499$ (1) Å). Rietveld analysis indicates that the crystal structure formed after heating in $\mathrm{Ar}$ at $850{ }^{\circ} \mathrm{C}$ is disordered.

\section{Ex situ formation of PtNi nanoalloys: PDF analysis}

In Fig. 14 PDF fit of the ex situ data collected after heat treatment of the precursor at $850{ }^{\circ} \mathrm{C}$ and cooling in the tube furnace is shown. The experimental interatomic distances are compared to the theoretical distances in Table S6 (ESI, $\dagger$ Section 6). PDF data were fitted against a disordered PtNi model and the

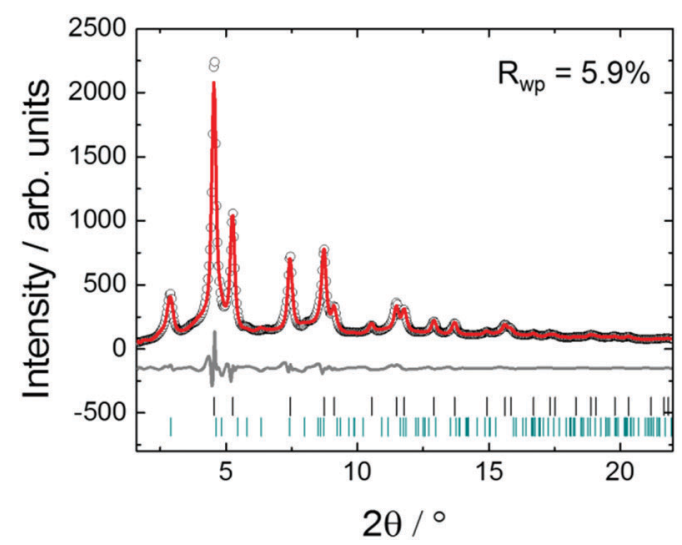

Fig. 13 Rietveld analysis of ex situ HR-XRPD data after heating at $850{ }^{\circ} \mathrm{C}$ and subsequent cooling in a tube furnace. The black circles represent the experimental data, while the red and the grey curves represent the calculated data and the difference, respectively. Tick marks represent the calculated positions of disordered PtNi (black) and carbon reflections (green). $R_{\mathrm{wp}}$ is the fit residual.

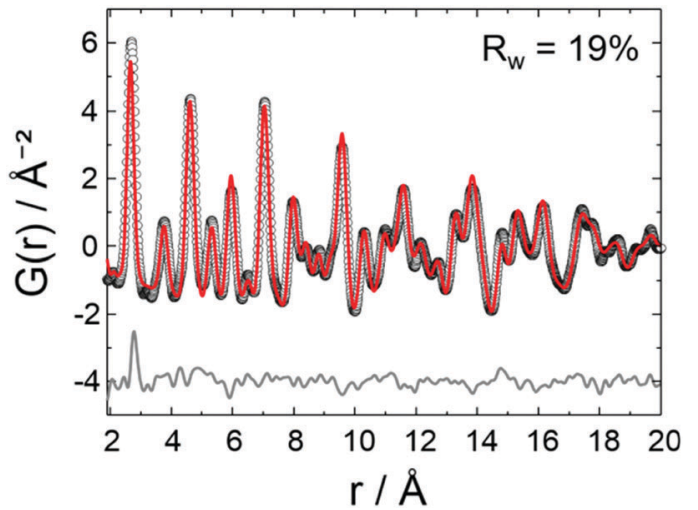

Fig. 14 PDF fit of ex situ data after heat treatment at $850{ }^{\circ} \mathrm{C}$ and subsequent cooling in a tube furnace: the black circles represent the experimental data, whereas the red and the grey curves represent the calculated data for the disordered model and the difference, respectively. $R_{\mathrm{w}}$ is the PDF fit residual.

results are provided in the ESI, $\dagger$ (Section 6, Table S7). The PDF fit in Fig. 14 shows that the local structure of PtNi nanoalloy is disordered after the conventional synthesis under $\mathrm{Ar}$ at $850{ }^{\circ} \mathrm{C}$ and subsequent cooling, as the fit residual $\left(R_{\mathrm{w}}\right)$ supports the good agreement between the disordered model and the experimental data (Fig. 14).

Fig. 15 shows the HAADF-STEM images of the precursor (Fig. 15a and b) and the ex situ synthesised sample (Fig. 15c and d). The particle size increases from 10-15 $\AA$ to $50-60 \AA$ in diameter after heat treatment at $850{ }^{\circ} \mathrm{C}$ and subsequent cooling in the tube furnace which is due to sintering at elevated temperatures (Fig. 15b and d). On the other hand, stability of the HGS support is proved as the average size and shape of the graphitic spheres seem to be preserved after heat treatment (Fig. 15a and c). Some precursor nanoparticles appeared to be crystalline under the microscope as the lattice fringes are clearly visible in Fig. 15b. Nonetheless, these images do not represent the entire sample as only a small fraction of the sample is imaged. In addition, room temperature (in situ) PDF data do not show features of a crystalline precursor.
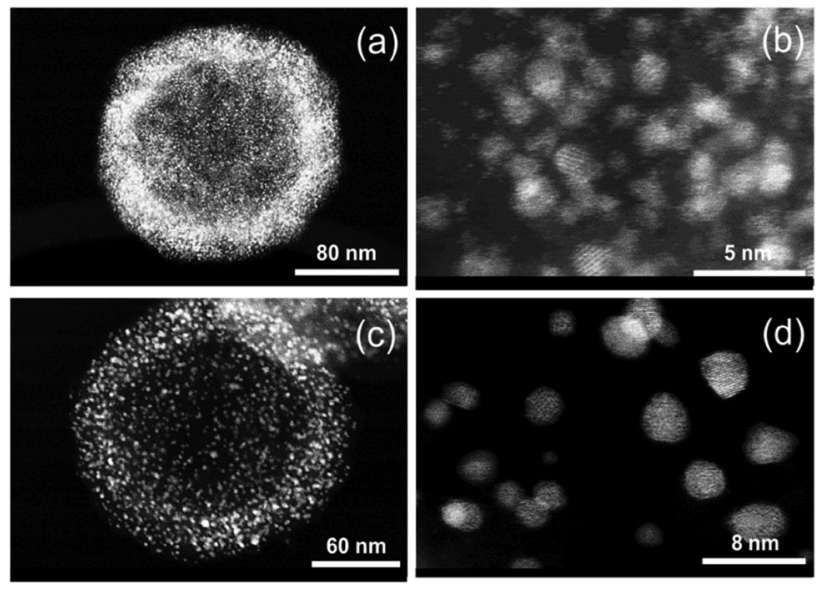

Fig. 15 HAADF-STEM images of PtNi on HGSs: ( $a$ and $b$ ) before and ( $c$ and $d$ ) after heating at $850{ }^{\circ} \mathrm{C}$ and subsequent cooling in a tube furnace. 


\section{Conclusions}

In this paper disordering/ordering mechanisms occurring during the formation of PtNi nanoalloys were directly probed by using the power of combined in situlex situ HR-XRPD and X-ray PDF analyses. During stepwise heating up to $800{ }^{\circ} \mathrm{C}$ the precursor transforms into a disordered PtNi nanoalloy. Then, upon subsequent cooling to $400{ }^{\circ} \mathrm{C}$, a partially ordered structure is obtained. The disordered nanoalloy requires time for restructuring. The atoms migrate slowly from their random positions to preferential positions in the unit cell to form an ordered nanoalloy. The alloy needs to be cooled relatively slowly to room temperature to achieve complete ordering. Upon rapid cooling only the disordered structure is obtained. Fitting of in situ/ex situ PDF data using different models provides detailed insights into the local structures of the PtNi nanoalloys formed.

Usually PtNi catalysts supported on HGS are prepared by heating in a furnace. For a respective sample (ex situ synthesised) no structural ordering was observed. In situ data collection at distinct temperatures requires more time than heating of the sample during conventional synthesis in a tube furnace. The studies underline that with respect to disordering/ordering of the alloy, temperature and time are decisive for the resulting structures. In this example, rapid cooling will not lead to the formation of an ordered alloy. Thus, in situ and ex situ experiments do not necessarily probe exactly the same state of a material and must be evaluated very carefully before general conclusions are drawn.

This work evidences the strength of the in situ PDF for the analysis of nanoparticles with very small (10-15 ̊) sizes. PDF provides direct insights into local structural changes under nonambient conditions. This information is crucial for the design of tailor-made catalysts for fuel cells or other applications.

\section{Conflicts of interest}

There are no conflicts to declare.

\section{Acknowledgements}

Authors thank Norbert Pfänder (Max-Planck Institute for Chemical Energy Conversion, Mülheim an der Ruhr) for HAADF-STEM analyses. The authors are indebted to Thomas Bernert (Max-PlanckInsitut für Kohlenforschung, Mülheim an der Ruhr) for the help during synchrotron measurements. Catherine Dejoie, Carlotta Giacobbe, Mauro Coduri and Andy Fitch (ID22 beamline at ESRF) are gratefully acknowledged for the technical support and discussion during the measurements as well as Dominik Daisenberger and Michael Wharmby (I15 beamline at DLS). Funding by BMWi under grant number 03ET6080B is gratefully acknowledged. The authors express their gratitude to the Max Planck Society for funding. Open Access funding provided by the Max Planck Society.

\section{References}

1 L. Xiong and A. Manthiram, J. Mater. Chem., 2004, 14, 1454-1460.
2 H. A. Gasteiger, S. S. Kocha, B. Sompalli and F. T. Wagner, Appl. Catal., B, 2005, 56, 9.

3 R. Bashyam and P. Zelenay, Nature, 2006, 443, 63-66.

4 H. A. Gasteiger and N. M. Marković, Science, 2009, 324, 48-49.

5 M. Debe, Nature, 2012, 486, 43-51.

6 M. Chiwata, H. Yano, S. Ogawa, M. Watanabe, A. Iiyama and H. Uchida, Electrochemistry, 2016, 84(3), 133-137.

7 A. Rabis, P. Rodriguez and T. J. Schmidt, ACS Catal., 2012, 2, 864-890.

8 S. Mukerjee, J. Appl. Electrochem., 1990, 20, 537-548.

9 J. C. Meier, C. Galeano, I. Katsounaros, J. Witte, H. J. Bongard, A. A. Topalov, C. Baldizzone, S. Mezzavilla, F. Schüth and K. J. J. Mayrhofer, Beilstein J. Nanotechnol., 2014, 5, 44-67.

10 D. He, H. Tang, Z. Kou, M. Pan, X. Sun, J. Zhang and S. Mu, Adv. Mater., 2017, 29, 1601741.

11 E. Antolini, J. R. C. Salgado, R. M. da Silva and E. R. Gonzales, Mater. Chem. Phys., 2007, 101, 395-403.

12 C. Wang, N. M. Markovic and V. R. Stamenkovic, ACS Catal., 2012, 2, 891-898.

13 K. Hyun, J. H. Lee, C. W. Yoon and Y. Kwon, Int. J. Electrochem. Sci., 2013, 8, 11752-11767.

14 C. Baldizzone, S. Mezzavilla, H. W. P. Carvalho, J. C. Meier, A. K. Schuppert, M. Huggen, C. Galeano, J.-D. Grunwaldt, F. Schüth and K. J. J. Mayrhofer, Angew. Chem., Int. Ed., 2014, 53, 14250-14254.

15 S. Shan, J. Luo, L. Yang and C.-J. Zhong, Catal. Sci. Technol., 2014, 4, 3570.

16 V. R. Stamenkovic, B. S. Mun, K. J. J. Mayrhofer, P. N. Ross and N. M. Markovic, J. Am. Chem. Soc., 2006, 128, 8813-8819.

17 V. Stamenkovic, B. S. Mun, K. J. J. Mayrhofer, P. N. Ross, N. M. Markovic, J. Rossmeisl, J. Greeley and J. K. Nørskov, Angew. Chem., Int. Ed., 2006, 45, 2897-2901.

18 L. Zou, J. Fan, Y. Zhou, C. Wang, J. Li, Z. Zou and H. Yang, Nano Res., 2015, 8(8), 2777-2788.

19 L. Dubau, M. Lopez-Haro, J. Durst and F. Maillard, Catal. Today, 2016, 262, 146-154.

20 O. Seo, J. Y. Lee, J. M. Kim, J.-W. Kim, H. C. Kang, J. Chung and D. Y. Noh, J. Alloys Compd., 2016, 666, 232-236.

21 T.-Y. Jeon, S. K. Kim, N. Pinna, A. Sharma, J. Park, S. Y. Lee, H. C. Lee, S.-W. Kang, H.-K. Lee and H. H. Lee, Chem. Mater., 2016, 28, 1879-1887.

22 Q. Huang, H. Yang, Y. Tang, T. Lu and D. L. Akins, Electrochem. Commun., 2006, 8, 1220-1224.

23 M. Nesselberger, S. Ashton, J. C. Meier, I. Katsounaros, K. J. J. Mayrhofer and M. Arenz, J. Am. Chem. Soc., 2011, 133, 17428.

24 N. M. Marković, Fuel Cells, 2001, 1, 105.

25 H. A. Gasteiger and N. M. Marković, Science, 2009, 324, 48-49. 26 S. Mezzavilla, C. Baldizzone, A.-C. Swertz, N. Hodnik, E. Pizzutilo, G. Polymeros, G. P. Keeley, J. Knossalla, M. Heggen, K. J. J. Mayrhofer and F. Schüth, ACS Catal., 2016, 6, 8058-8068.

27 C. Galeano, J. C. Meier, V. Peinecke, H. Bongard, I. Katsounaros, A. A. Topalov, A. Lu, K. J. J. Mayrhofer and F. Schüth, J. Am. Chem. Soc., 2012, 134, 20457-20465.

28 S. Mukerjee, S. Srinivasan and M. P. Soriaga, J. Electrochem. Soc., 1995, 142(5), 1409-1422. 
29 P. J. Chupas, K. W. Chapman, H. Chen and C. P. Grey, Catal. Today, 2009, 145, 213.

30 E. L. Redmond, B. P. Setzler, P. Juhas, S. J. L. Billinge and T. F. Fuller, Electrochem. Solid-State Lett., 2012, 15(5), B72-B74.

31 V. Petkov, B. Prasai, S. Shan, Y. Ren, J. Wu, H. Cronk, J. Luo and C.-J. Zhong, Nanoscale, 2016, 8(20), 10749-10767.

32 X. Tuaev, S. Rudi, V. Petkov, A. Hoell and P. Strasser, ACS Nano, 2013, 7(7), 5666-5674.

33 V. Petkov, S. J. L. Billinge, J. Heising, M. G. Kanatzidis, S. D. Shastri and S. Kycia, Mater. Res. Soc. Symp. Proc., 2000, 590, 151-156.

34 S. J. L. Billinge, Z. Kristallogr., 2004, 219, 117-121.

35 U. Esch and A. Schneider, Z. Elektrochem. Angew. Phys. Chem., 1944, 50, 268-274.

36 J. C. Woolley and B. Bates, J. Less-Common Met., 1960, 2, 11-18.
37 S. Mezzavilla, C. Baldizzone, K. J. J. Mayrhofer and F. Schüth, ACS Appl. Mater. Interfaces, 2015, 7, 12914-12922.

38 J. Knossalla, D. Jalalpoor and F. Schüth, Chem. Mater., 2017, 29, 7062-7072.

39 A. P. Hammersley, S. O. Svensson, M. Hanfland, A. N. Fitch and D. Hausermann, High Pressure Res., 1996, 14, 235-248.

40 A. A. Coelho, TOPAS Academic version 4.2, Coelho Software, 2009.

41 P. Juhás, T. Davis, C. L. Farrow and S. J. L. Billinge, J. Appl. Crystallogr., 2013, 46, 560-566.

42 C. L. Farrow, P. Juhas, J. W. Liu, D. Bryndin, E. S. Božin, J. Bloch, Th. Proffen and S. J. L. Billinge, J. Phys.: Condens. Matter, 2007, 19, 335219.

43 K. Brandenburg and H. Putz, Diamond Version 3.2k, Bonn, 2014. 44 C. Leroux, M. C. Cadeville, V. Pierron-Bohnes, G. Inden and F. Hinz, J. Phys. F: Met. Phys., 1988, 18, 2033-2051.

45 A. W. Hull, Phys. Rev., 1917, 10, 661-696. 